\title{
Soliton dynamics at an interface between a uniform medium and a nonlinear optical lattice
}

\author{
Fatkhulla Kh. Abdullaev, ${ }^{1, *}$ Ravil M. Galimzyanov, ${ }^{1}$ Marijana Brtka, ${ }^{2}$ and Lauro Tomio ${ }^{3}$ \\ ${ }^{1}$ Physical-Technical Institute of the Academy of Sciences, G. Mavlyanov Street 2-b, 100084 Tashkent, Uzbekistan \\ ${ }^{2}$ Instituto de Física, Universidade de São Paulo, CP 66318, São Paulo 05315-970, SP, Brazil \\ ${ }^{3}$ Instituto de Física Teórica, São Paulo State University (UNESP), Rua Pamplona 145, São Paulo 01405-900, SP, Brazil
}

(Received 28 January 2009; published 19 May 2009)

\begin{abstract}
We study trapping and propagation of a matter-wave soliton through the interface between uniform medium and a nonlinear optical lattice. Different regimes for transmission of a broad and a narrow solitons are investigated. Reflections and transmissions of solitons are predicted as a function of the lattice phase. The existence of a threshold in the amplitude of the nonlinear optical lattice, separating the transmission and reflection regimes, is verified. The localized nonlinear surface state, corresponding to the soliton trapped by the interface, is found. Variational approach predictions are confirmed by numerical simulations for the original Gross-Pitaevskii equation with nonlinear periodic potentials.
\end{abstract}

DOI: 10.1103/PhysRevE.79.056220

PACS number(s): 05.45.- a, 03.75.Lm, 05.45.Yv, 02.30.Jr

\section{INTRODUCTION}

Investigation of processes of reflection, transmission, and trapping of a nonlinear wave packet at the interface between two different nonlinear media represents one of the fundamental problems of the nonlinear physics $[1,2]$. Recently, the problem of reflection and/or transmission of a soliton at the interface between a nonlinear uniform media and a linear periodic structure, under the conditions of the Bragg resonance, has been considered in Ref. [3]. There it was shown the possibility of controlling such a structure in the regime of the soliton mirror.

Considering the actual experimental possibilities, it will be also of interest to study the transmission and trapping phenomena when we have a periodic variation in space of the parameter related to the nonlinearity. Such system can be realized in Bose-Einstein condensates (BECs) by using a periodically modulated in space external magnetic field or optically induced Feshbach resonances [4-6]. Standing optical wave can induce in BEC periodic space modulation in the atom-atom scattering length. In the Gross-Pitaevskii (GP) equation it leads to periodic space modulations of the mean field nonlinearity; i.e., producing a nonlinear optical lattice (NOL) [7-15]. Considering the two-component case in the one-dimensional (1D) limit, the properties of BEC confined in NOL, as well as existence of soliton solutions and their stability, are investigated in Ref. [16]. BECs with finite segment of periodically space-modulated atomic scattering length (shallow optical lattice) are considered in Ref. [17], where matter-wave optical limits and bistability are predicted. For the dynamics of matter-wave propagation under different conditions, see the review in [18] and references therein. Gap solitons are analyzed in Ref. [19], where it was shown that localized nonlinear wave packets can exist in NOL for attractive condensates as well as for repulsive ones. The stability analysis showed that the bright solitons are stable in a very narrow region of parameters [20]. However, such analysis is absent in case of the existence of an inter-

\footnotetext{
*Corresponding author; fatkhulla@yahoo.com
}

face. Linear surface states in lattices with management of the diffraction have been recently considered in Ref. [21].

In the present paper we consider regimes of reflection, transmission, and trapping of a matter-wave soliton incident on the interface between uniform medium and a nonlinear optical lattice. Particular attention will be devoted to the possible existence of nonlinear surface states for matter waves. An interface induces changes in the effective potentials for the soliton center and width and can create a surface soliton. Also the stability can be enhanced. The dynamics of a BEC in a quasi-one-dimensional elongated trap will be treated by considering the GP formalism reduced to the 1D space limit.

Recently, an investigation done in Ref. [22] considered two-dimensional nonlinear surface states (surface solitons) at an interface in a superposition of a periodic potential and periodic modulations of the nonlinear space parameter. The physical system is motivated by optical structures writing on quartz by femtosecond laser (fs laser). In this case, variations in the Kerr nonlinearity remain of the same sign and are out of the phase with the periodic variations in the linear refractive index. As opposed to this nonlinear optical system, in BEC case we can also realize the cases of periodic modulations when the nonlinearity changes sign.

The transmission characteristics of solitons are defined by the effective potential induced by the interface and nonlinear periodic lattice. The effective potential strongly depends on the soliton parameters. Unlike the soliton transmission through the linear lattice, in the case of nonlinear periodic lattice we have nontrivial intensity dependence (number of atoms) for the form of the potential relief as well as a threshold behavior depending on the amplitude of the nonlinearity modulations in space. It means that by change in the amplitude of modulations performed by variation in external magnetic field near the Feshbach resonance point, we can form a mirror for the matter-wave solitons, selecting the solitons by the number of atoms.

The paper is organized as follows. In Sec. II the model is formulated and variational equations for the soliton parameters are derived. In Sec. III characteristics of stationary soliton trapped by interface are investigated. The reflection and transmission regimes for narrow and broad solitons are analyzed. The summary of obtained results is given in Sec. IV. 


\section{MODEL}

In order to describe the propagation of a matter-wave soliton in the elongated quasi-1D condensate with attractive interaction, we consider the GP equation in a 1D space approach where the physical space-time variables are given by $(\bar{x}, \bar{t})$, and the corresponding dimensionless variables are $(x, t)$ :

$$
i \hbar \frac{\partial \psi}{\partial \bar{t}}+\frac{\hbar^{2}}{2 m} \frac{\partial^{2} \psi}{\partial \bar{x}^{2}}+g_{1 \mathrm{D}}(\bar{x})|\psi|^{2} \psi=0,
$$

where $\psi \equiv \psi(\bar{x}, \bar{t})$ and $g_{1 \mathrm{D}}(\bar{x}) \equiv 2 \hbar a_{s}(\bar{x}) \omega_{\perp}$. Here, $\omega_{\perp}$ is the transverse frequency of the trap and $a_{s}(\bar{x})$ is the spatially dependent atomic scattering length, which is supposed to vary in space for $\bar{x}>0$ as $a_{s}(\bar{x})=a_{0}+\theta(\bar{x})\left[\delta_{0}+a_{1} \sin (2 k \bar{x})\right]$. Here, $\theta(x)=0(1)$ for $x<0(x>0), \delta_{0}$ is a constant, $a_{0}$ is the natural two-body scattering length, and the wave number $k$ is related to the lattice period $L$ by $k \equiv 2 \pi / L$. The number of atoms $\bar{N}$ normalizes the wave function as

$$
\bar{N}=\int_{-\infty}^{\infty}|\psi|^{2} d \bar{x}
$$

To avoid the collapse in the attractive BEC, the condition $\left|a_{s}\right| \bar{N} / \omega_{\perp}<0.676$ should be satisfied [23].

The transformation to the new set of dimensionless spacetime variables $(x, t)$ is given by the following:

$$
\begin{gathered}
x \equiv k \bar{x}, \quad t \equiv \omega_{R} \bar{t}, \quad \gamma(x) \equiv \frac{a_{s}(\bar{x})}{\left|a_{0}\right|}, \quad \Delta_{0} \equiv \frac{\delta_{0}}{\left|a_{0}\right|}, \\
E_{R} \equiv \hbar \omega_{R} \equiv \frac{\hbar^{2} k^{2}}{2 m}, \quad u \equiv u(x, t) \equiv \sqrt{\frac{2 \omega_{\perp}}{\omega_{R}}\left|a_{0}\right| \psi} .
\end{gathered}
$$

With the above, where $E_{R}$ is the recoil energy, we obtain the dimensionless form of the 1D GP equation

$$
\begin{gathered}
i u_{t}+u_{x x}+\gamma(x)|u|^{2} u=0, \\
\gamma(x)=\left\{\gamma_{0}+\theta(x)\left[\Delta_{0}+\gamma_{1} \sin (2 x)\right]\right\},
\end{gathered}
$$

where $\gamma_{0}=a_{0} /\left|a_{0}\right|= \pm 1$ (for the attractive and repulsive condensates, respectively) and $\gamma_{1}=a_{1} /\left|a_{0}\right|$. In the above equation and in the following, we use the abbreviated notation for partial differential equations, such that $u_{t} \equiv \partial u / \partial t$. The normalization of $u$ and $N$ relates to the number of atoms $\bar{N}$, which is conserved. From Eqs. (2) and (3), we obtain

$$
N=\int_{-\infty}^{\infty}|u|^{2} d x=\frac{4 m\left|a_{0}\right| \omega_{\perp}}{\hbar k} \bar{N}
$$

Below we will consider the evolution of bright solitons $\left(\gamma_{0}\right.$ $=1)$. When solitons collide at the interface $(x=0)$, different scenarios are possible resulting in reflection, transmission, or trapping. Let us consider different limiting cases of broad and narrow solitons (with respect to the period of modulations). To study the soliton evolution we shall use the variational approach (VA) [24]. According to this method we should calculate an averaged Lagrangian, and then, using the
Euler-Lagrange equations, obtain the equations for the soliton parameters.

The Lagrangian density corresponding to Eq. (3) is given by

$$
L=\frac{i}{2}\left(u_{t} u^{\star}-u_{t}^{\star} u\right)-\left|u_{x}\right|^{2}+\frac{1}{2} \gamma(x)|u|^{4}
$$

In deriving our variational model we proceed from the following ansatz for a soliton:

$$
u=\sqrt{2} A \operatorname{sech}\left(\frac{x-\xi}{\alpha}\right) e^{i \beta(x-\xi)^{2}+i \kappa(x-\xi)+i \phi} .
$$

In order to obtain the equations for the soliton parameters $(A, \alpha, \beta, \kappa, \xi, \phi)$, we calculate the averaged Lagrangian $\bar{L}$ $=\int_{-\infty}^{\infty} L(x, t) d x$ with the above trial function [Eq. (6)]:

$$
\begin{aligned}
\frac{\bar{L}}{N}= & -\frac{\pi^{2}}{12} \beta_{t} \alpha^{2}+\kappa \xi_{t}-\phi_{t}-\frac{1}{3 \alpha^{2}}-\frac{\pi^{2}}{3} \beta^{2} \alpha^{2}-\kappa^{2}+\frac{N \gamma_{0}}{6 \alpha} \\
& +\frac{\Delta_{0} N}{8 \alpha} F_{0}(\xi, \alpha)+\frac{\gamma_{1} N}{8 \alpha} F_{2}(\xi, \alpha),
\end{aligned}
$$

where

$$
\begin{gathered}
F_{0}(\xi, \alpha)=\frac{2}{3}+\tanh \left(\frac{\xi}{\alpha}\right)-\frac{1}{3} \tanh ^{3}\left(\frac{\xi}{\alpha}\right), \\
F_{2}(\xi, \alpha)=\int_{-\xi / \alpha}^{\infty} \operatorname{sech}^{4}(z) \sin (2 z \alpha+2 \xi) d z
\end{gathered}
$$

with the integration variable $z=(x-\xi) / \alpha$. The EulerLagrange equations lead to the following:

$$
\begin{gathered}
\alpha_{t}=4 \alpha \beta, \quad \xi_{t}=2 \kappa \\
\kappa_{t}=\frac{N}{8 \alpha} \frac{\partial}{\partial \xi}\left(\Delta_{0} F_{0}+\gamma_{1} F_{2}\right), \\
\beta_{t}=-4 \beta^{2}+\frac{4}{\pi^{2} \alpha^{4}}-\frac{N \gamma_{0}}{\pi^{2} \alpha^{3}}+\frac{3 N}{4 \pi^{2} \alpha} \frac{\partial}{\partial \alpha}\left(\frac{\Delta_{0} F_{0}+\gamma_{1} F_{2}}{\alpha}\right) .
\end{gathered}
$$

Eliminating the parameter $\beta$ from the equations, we get the following evolution equation for the width $\alpha$ :

$$
\alpha_{t t}=\frac{16}{\pi^{2} \alpha^{3}}-\frac{4 N \gamma_{0}}{\pi^{2} \alpha^{2}}+\frac{3 N}{\pi^{2}} \frac{\partial}{\partial \alpha}\left(\frac{\Delta_{0} F_{0}+\gamma_{1} F_{2}}{\alpha}\right) .
$$

This equation can be rewritten as

$$
\alpha_{t t}=-\frac{\partial V_{\alpha}(\alpha, \xi)}{\partial \alpha}
$$

where

$$
V_{\alpha}(\alpha, \xi)=\frac{8}{\pi^{2} \alpha^{2}}-\frac{N}{\pi^{2} \alpha}\left[4 \gamma_{0}+3\left(\Delta_{0} F_{0}+\gamma_{1} F_{2}\right)\right] .
$$

In a similar way for the soliton center we get 

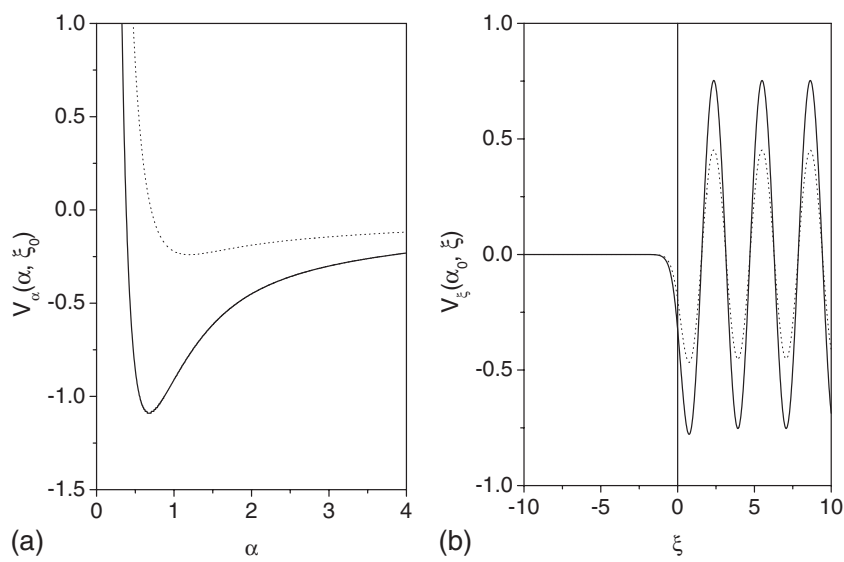

FIG. 1. Profiles of the effective potentials $V_{\alpha}\left(\alpha, \xi_{0}\right)$ [as a function of the width $\alpha$, shown in (a)] and $V_{\xi}\left(\alpha_{0}, \xi\right)$ [as a function of $\xi$, shown in (b)], where values of $\alpha_{0}$ and $\xi_{0}$ correspond to the stationary point of the set of Eqs. (12) and (15). Vertical line in (b) indicates the interface. Two cases are depicted, $N=1.5$ with $\alpha_{0}=1.213, \xi_{0}=0.668$ (dotted line) and $N=2.5$ with $\alpha_{0}=0.674$, $\xi_{0}=0.773$ (solid line). Other parameters are $\gamma_{0}=1, \gamma_{1}=1.2$, and $\Delta_{0}=0$.

$$
\xi_{t t}=-\frac{\partial V_{\xi}(\alpha, \xi)}{\partial \xi},
$$

where

$$
V_{\xi}(\alpha, \xi)=-\frac{N}{4 \alpha}\left(\Delta_{0} F_{0}+\gamma_{1} F_{2}\right)
$$

Typical profiles of effective potentials $V_{\alpha}(\alpha, \xi)$ and $V_{\xi}(\alpha, \xi)$ for the case of narrow solitons are shown in Fig. 1. The stationary point given by $\alpha_{0}$ and $\xi_{0}$ is obtained in a selfconsistent manner using Eqs. (14) and (16). It should be noted that when the soliton norm decreases (increase in the width), the amplitude of the potential $V_{\xi}$ decreases. Accurate approximative analytical expressions for the effective potentials $V_{\alpha}(\alpha, \xi)$ and $V_{\xi}(\alpha, \xi)$, valid in a wide range of variables, $\alpha$ and $\xi$, can be obtained using the asymptotic representation of the integral [Eq. (8)] for $F_{2}(\xi, \alpha)$ :

$$
\begin{aligned}
F_{2}(\xi, \alpha)= & g(\alpha) \operatorname{sech}^{4}(\xi / \alpha) \\
& +\frac{2\left(1+\alpha^{2}\right) \pi \alpha \sin (2 \xi)}{3 \sinh (\pi \alpha)}[1+\tanh (\xi / \alpha)],
\end{aligned}
$$

where $g(\alpha) \equiv F_{2}(0, \alpha)$. This expression is obtained by sewing two different approximations for the integral, valid at the ranges: $-\infty<\xi \ll-A, A \gg 1$, and $a \ll \xi<\infty$. The resulting interpolating formula describes well the integral $F_{2}$ in all regions of $\xi$, including the region $0<\xi<a$. The dependence of the factor $g(\alpha)$ on the soliton width is shown in Fig. 2.

\section{RESULTS}

All calculations below are performed for a solitary matter wave with background nonlinearity $\gamma_{0}=1$ and $\Delta_{0}=0$. In all the simulations of the soliton transmission and reflection, the incident wave packets are taken in the standard soliton form,

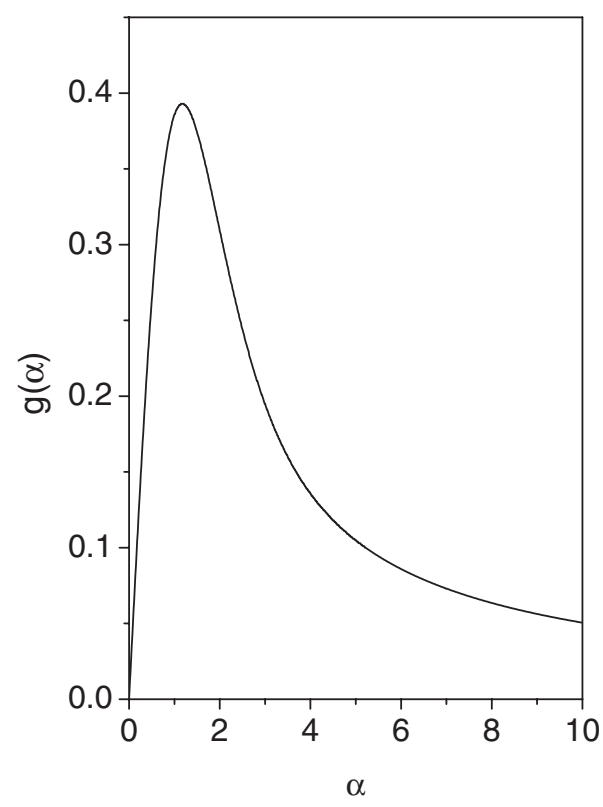

FIG. 2. Factor $g(\alpha)$ versus the soliton width $\alpha$.

with the initial parameters given by $A=N / 4, \alpha_{0}=1 / A, \beta=0$, and $\phi=0$. In the starting position, the soliton is in a homogeneous medium.

\section{A. Stationary soliton}

A stationary solution, within a semi-infinite lattice, is given by expression

$$
u_{0}(x)=\sqrt{2} A \operatorname{sech}\left(\frac{x-\xi_{0}}{\alpha_{0}}\right),
$$

where the stationary values, for the soliton position $\xi$ and width $\alpha$, are obtained in a self-consistent manner from the set of equations

$$
\begin{aligned}
& \left.\frac{\partial V_{\xi}(\alpha, \xi)}{\partial \xi}\right|_{\alpha_{0}, \xi_{0}}=0, \\
& \left.\frac{\partial V_{\alpha}(\alpha, \xi)}{\partial \alpha}\right|_{\alpha_{0}, \xi_{0}}=0 .
\end{aligned}
$$

Figure 3 depicts stationary values of the soliton width $\alpha_{0}$ and its position $\xi_{0}$ versus the norm $N$.

As seen from Fig. 3(b), the position of the soliton center shifts from zero to some fixed-point value as the number of atoms increases. In order to check the stationary solution, which was obtained above, we solve directly the GrossPitaeskii equation [Eq. (3)] with the starting wave packet [Eq. (18)]. The results of the full partial differential equations (PDE) computation at $t=60$ are given in Fig. 4 . The parameters we consider are $N=2.5, \gamma_{0}=1$, and $\gamma_{1}=1.2$. In this case, the stationary point obtained from the variational equations corresponds to $\alpha_{0}=0.6083$ and $\xi_{0}=0.7730$. By starting with this variational point, the results of the full PDE simulation evolves to $\alpha_{0}=0.6293$ and $\xi_{0}=0.7778$ at $t=60$. Establishment of the stationary value of the soliton width in this case is 

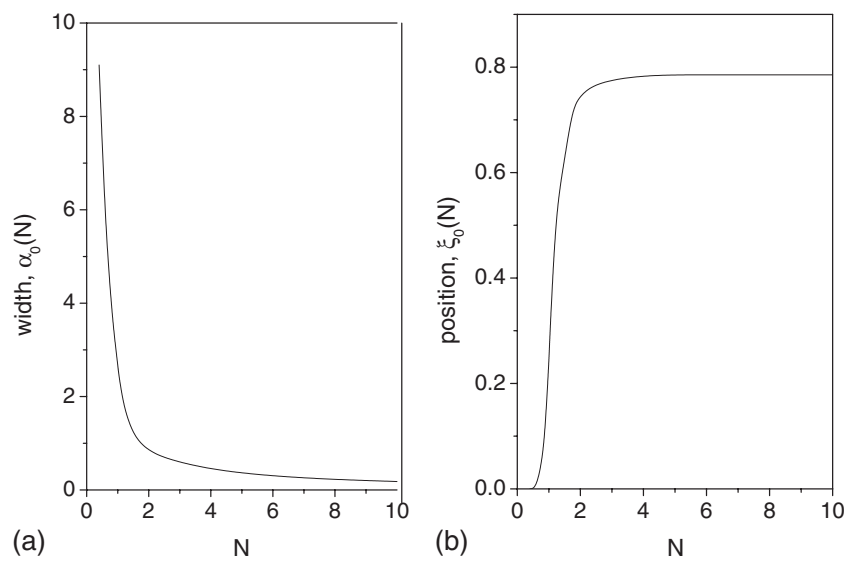

FIG. 3. Dependences of the (a) width $\alpha_{0}$ and (b) position $\xi_{0}$ of the stationary points on the norm $N$ for the case $\gamma_{1}=1.2$.

shown in Fig. 5 for the evolution of the wave packet.

We have also calculated the oscillation frequencies at the stationary point of the potentials $V_{\alpha}(\alpha, \xi)$ and $V_{\xi}(\alpha, \xi)$ versus the NOL strength $\gamma_{1}$ for the case $N=2.5\left(\alpha_{0}=1.6\right)$. The results of our calculations in the frame of the variational Eqs. (12) and (15) and PDE simulations are depicted in Fig. 6. One can see satisfactory agreement between variational and PDE results.

\section{B. Reflection and transmission of narrow solitons}

Reflection and transmission of solitons at the interface between a homogeneous medium and the optical lattice can be described by Eq. (15) considering the soliton as a particle

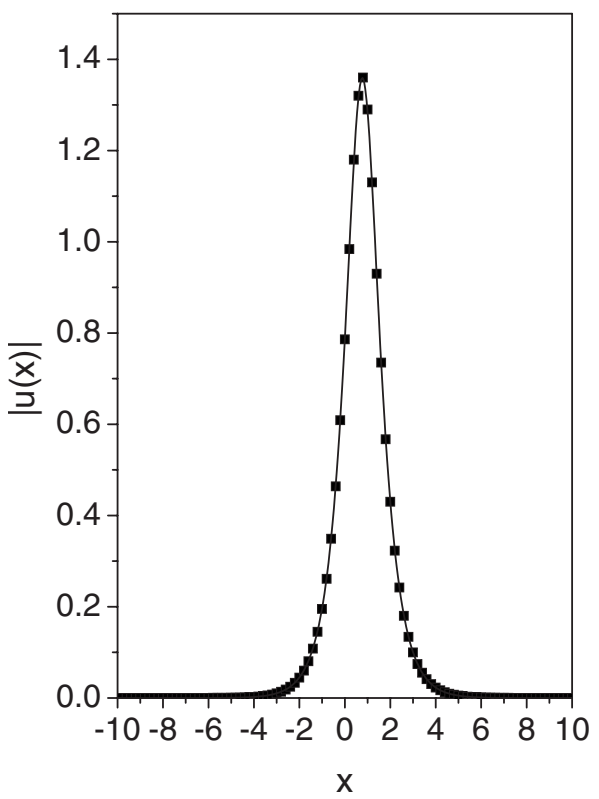

FIG. 4. The established stationary wave-packet profile $|u(x)|$ at $t=60$. Solid line stands for the full PDE results and squares are for the single soliton solution given by Eq. (6). The parameters are $N$ $=2.5, \gamma_{1}=1.2$. The stationary point corresponds to $\alpha_{0}=0.6083$ and $\xi_{0}=0.7730$ for the variational approach and $\alpha_{0}=0.6293$ and $\xi_{0}$ $=0.7778$ for the full PDE calculation at $t=60$.

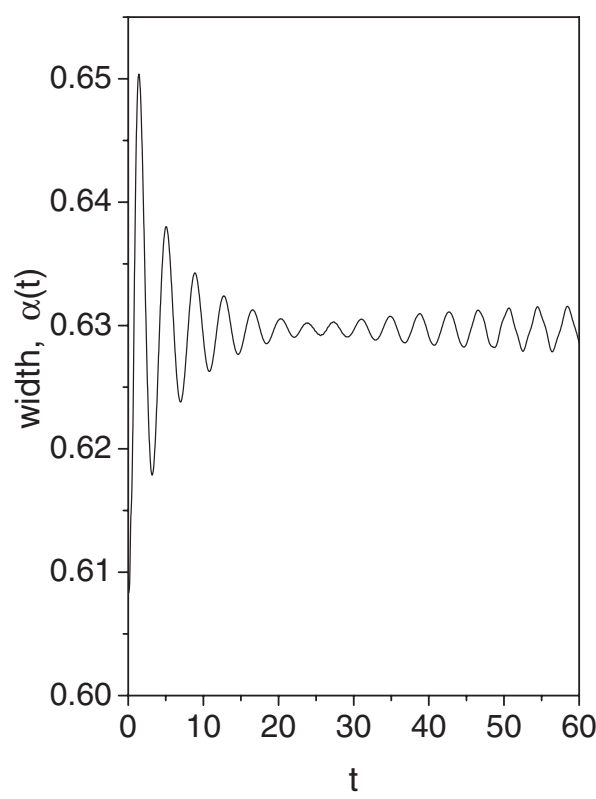

FIG. 5. Evolution of the wave-packet width in the course of establishment of stationarity when starting from the single soliton solution given by Eq. (6). The parameters are $N=2.5, \alpha_{0}$ $=0.6083, \quad \gamma_{1}=1.2$.

traveling under the effective potential $V_{\xi}(\alpha, \xi)$. Then, the condition of reflection or transmission is determined by the potential barrier height (see Fig. 1). In Fig. 7 we present simulations for transmission and reflection of the soliton at the interface, above and below the threshold value of the nonlinearity strength $\gamma_{1}$. The results obtained with ODE calculations, based on Eq. (15), are shown with dotted lines. The solid lines correspond to PDE simulations obtained from the GP Eq. (3). The soliton parameters are $\alpha_{0}=0.25, N=16$, and $v=1$, where $v \equiv \xi_{t}=2 \kappa$ is the soliton velocity [see Eq. (9)]. The potential barrier heights, $\gamma_{1}=0.022$, indicated in Fig. 7(a) and $\gamma_{1}=0.04$, indicated in Fig. 7(b), are, respectively, below and above the kinetic energy of the soliton, $E_{k i n}=v^{2} / 2$. As seen, the transmission and reflection condi-
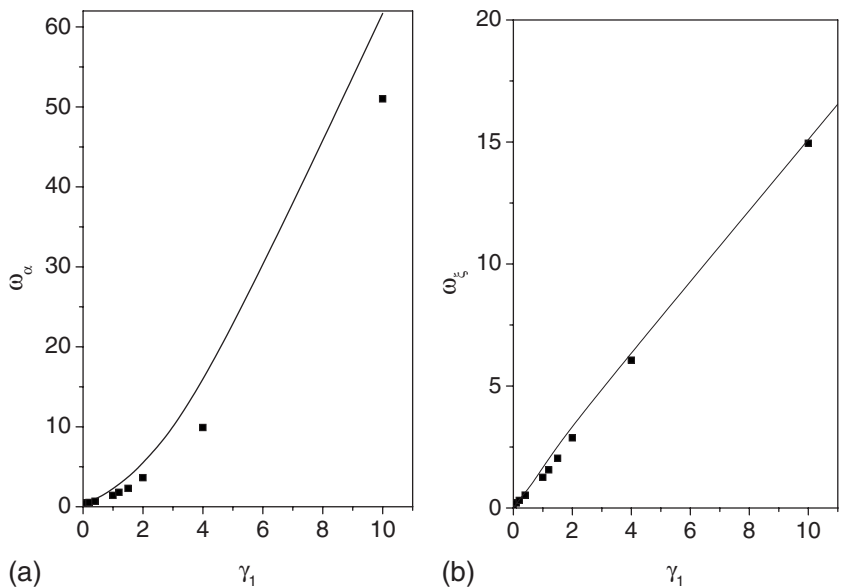

FIG. 6. Frequencies $\omega_{\alpha}$ and $\omega_{\xi}$ versus the potential strength $\gamma_{1}$ With solid lines we show results obtained with the variational approach. The full PDE results are shown with squares. The soliton norm is $N=2.5$. 

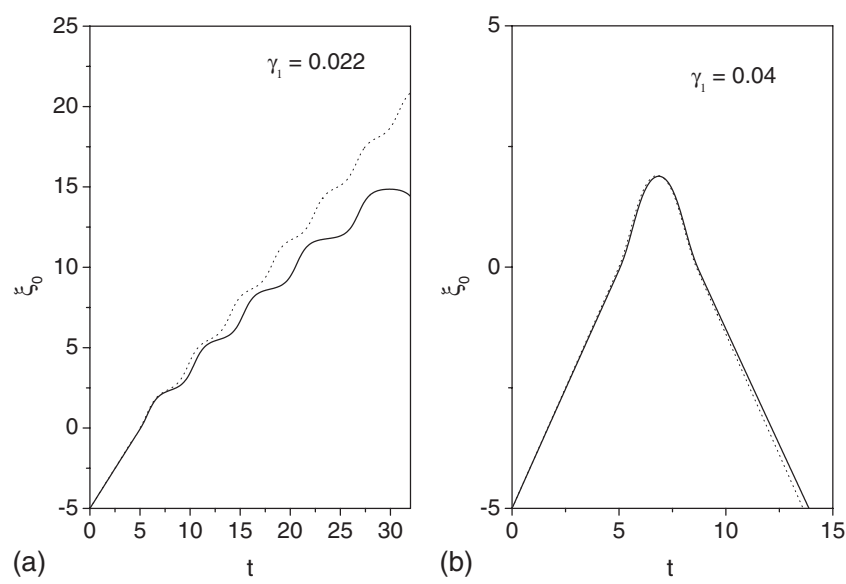

FIG. 7. Reflection and transmission of a soliton (a) below and (b) above the threshold value of $\gamma_{1}=0.023$. Solid lines stand for PDE simulations and dotted lines for variational ODE calculations. The parameters of the soliton are $\alpha_{0}=0.25, N=16$, and $v=1$.

tions obtained in this case are in a good agreement with the PDE simulations of Eq. (3). We also observe that the true soliton dynamics deviates from the analytical prediction for quite large times. At some depth of the soliton penetration into the optical lattice, the soliton can be trapped due to the radiation effects.

To study numerically the traveling of a narrow soliton in a wide range of strengths of the optical lattice $\left(0.022<\gamma_{1}\right.$ $<0.4$ ), we carried out corresponding PDE simulations presented in Fig. 8. The soliton parameters were $\alpha=0.25, N$ $=16$, and $v=1$. One can observe two regions of the optical lattice strength $\gamma_{1}$ which provide trapping of the soliton: $\gamma_{1}$ $\leq 0.023$ and $\gamma_{1} \geq 0.335$. The first region $\left(\gamma_{1} \leq 0.023\right)$ corre-

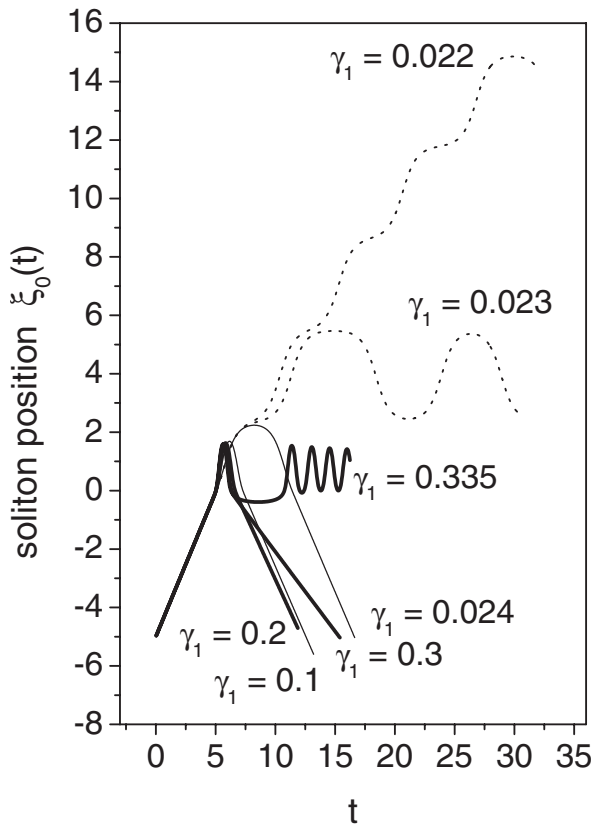

FIG. 8. Traveling of the position of a narrow soliton at different strengths of the periodic potential. Processes of transmission, reflection, and trapping are depicted. The soliton parameters are $\alpha_{0}$ $=0.25, N=16$, and $v=1$.

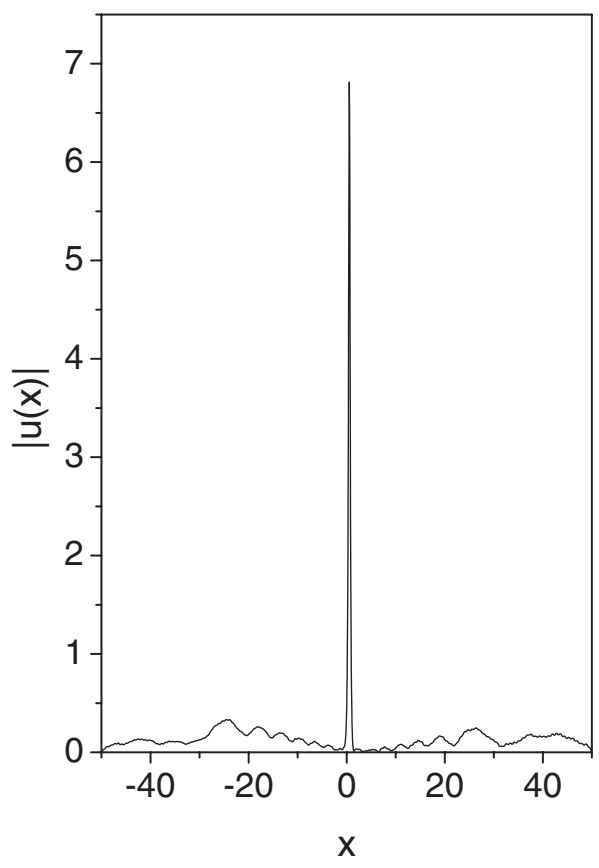

FIG. 9. Trapped soliton at $t=10$. The simulation is started from the single soliton solution given by Eq. (6) moving with velocity $v=1$. Its initial position is $\xi_{0}=-5$. Other parameters are $N=16, \gamma_{0}$ $=1, \gamma_{1}=0.4$, and $\alpha_{0}=0.25$.

sponds to the soliton motion above the barrier. The trapping in this case is caused by an unavoidable radiation, which decreases the soliton kinetic energy, in the course of its motion in the optical lattice. The cause of the soliton trapping in the second case $\left(\gamma_{1} \geq 0.335\right)$ can be explained by rearrangements of the wave packet due to deepening of the effective potential well, accompanied by strong radiation that results in transformation of the incident moving soliton to the stationary one. Typical profile of the trapped soliton in this case is depicted in Fig. 9. The evolution of the soliton wavepacket profile in the course of transmission of the optical lattice is presented in Fig. 10. As the soliton penetrates in the optical lattice, we observe that the amplitude of the transmitted soliton decreases due to noticeable radiation.

\section{Broad soliton}

Let us describe the dynamics of the broad soliton by Eqs. (12) and (15). The VA works well for the soliton propagation with the width less or of the order of the lattice period. The validity of VA for the dynamics of a broad (with respect to the lattice period) soliton should be checked by direct numerical simulations. As a rule one can expect a good agreement for the VA if radiation effects in propagation of the soliton in periodically modulated media are small [25]. As shown in Ref. [9], radiative effects at motion in the media with spatially periodic nonlinearity are small at the propagation of soliton with small velocity. The approximated expressions for the effective potentials $V_{\alpha}(\alpha, \xi)$ and $V_{\xi}(\alpha, \xi)$ can be simplified when considering large $\alpha$. So, as the soliton width grows to large $\alpha$, the second term of Eq. (17) can be neglected. By also imposing $\Delta_{0}=0$, the effective potential $V_{\xi}(\alpha, \xi)$ takes the form 


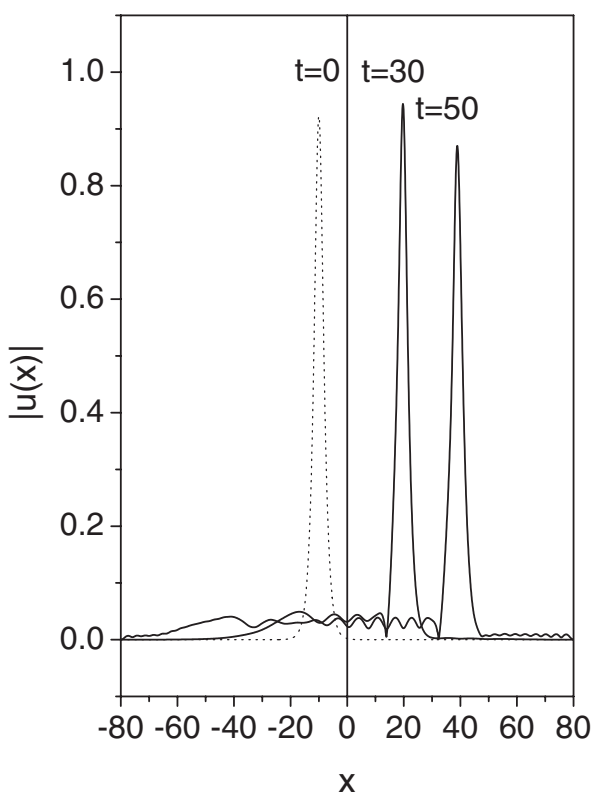

FIG. 10. Transmission of the narrow wave packet when starting from the single soliton solution with the parameters $N=2.721, \alpha_{0}$ $=1.47, \xi_{0}=-10, v=1$. The nonlinear optical lattice strength is $\gamma_{1}=0.1$.

$$
V_{\xi}(\alpha, \xi)=-\frac{N \gamma_{1}}{4} \frac{g(\alpha)}{\alpha} \operatorname{sech}^{4}(\xi / \alpha)
$$

For $\gamma_{1}>0$, the potential $V_{\xi}(\alpha, \xi)$ is a potential well, and for $\gamma_{1}<0$, the potential $V_{\xi}(\alpha, \xi)$ is a potential barrier. It means that the reflection of the soliton becomes only possible provided that $\gamma_{1}<0$. The sign of $\gamma_{1}$ is defined by the phase $\delta$ of the periodic modulation of the nonlinearity $\sim \sin (2 x+\delta)$. Thus, with the variation in such phase one can switch the matter-wave soliton from a transmission regime to a reflection one. The transmission (reflection) of the soliton occurs when the soliton kinetic energy is greater (smaller) than the potential barrier height. The threshold kinetic energy, $E_{c r}$, is given by

$$
E_{c r}=\frac{v^{2}}{2}=\left|V_{\xi}(\alpha, \xi)\right|_{\max }=N\left|\gamma_{1}\right| \frac{g(\alpha)}{4 \alpha} .
$$

Figure 11 depicts transmission of a broad soliton for the case $\gamma_{1}>0$ when the effective potential $V_{\xi}(\alpha, \xi)$ is a potential well and reflection and transmission of the soliton for the case $\gamma_{1}<0$ when the effective potential is a barrier. As seen, the conditions of transmission and reflection are well described by Eq. (22). It should be noted that trapping of the soliton is not observed since the effective potential $V_{\xi}(\alpha, \xi)$ is a short-range one, such that, far from the interface, motion of the soliton can be considered as free.

Let us discuss the possible effects which can be predicted in experiments with BEC in a cigar-type trap. As an example, we can consider the ${ }^{7} \mathrm{Li}$ condensate in the elongated trap with the transverse frequency $\omega_{\perp} \approx 2 \pi \times 10^{3} \mathrm{~Hz}$ and the longitudinal frequency $\omega_{x} \approx$ few $\mathrm{Hz}$. The density is $n$ $\approx 10^{9} \mathrm{~m}^{-1}$. The healing length and speed of sound are $\xi$ $\approx 2 \mu \mathrm{m}$ and $c \approx 5 \mathrm{~mm} / \mathrm{s}$. In a typical experiment [26] we

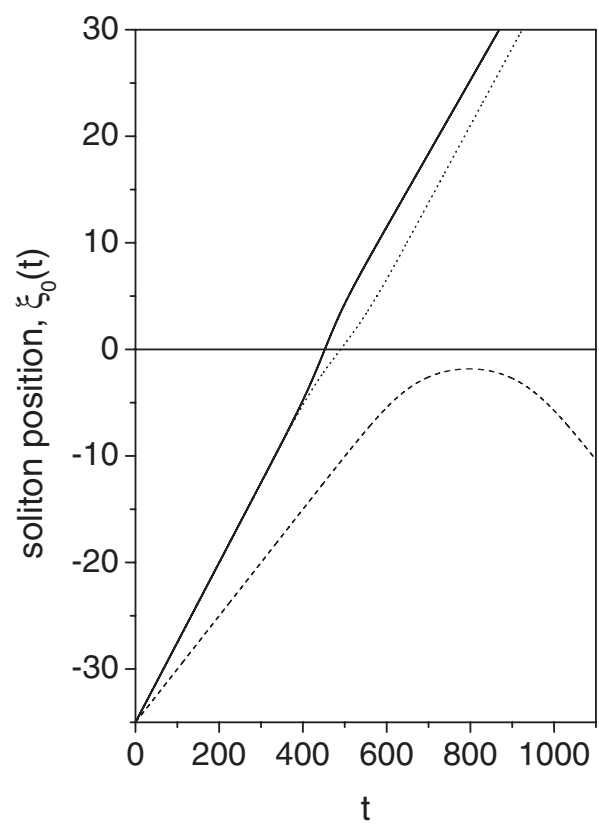

FIG. 11. Numerical simulations of reflection and transmission of a broad soliton with the parameters $N=0.6$ and $\alpha_{0}=6.667$. The solid line stands for the case $\gamma_{1}>0$ when the effective potential $V_{\xi}(\alpha, \xi)$ is a potential well. Dotted and dashed lines are for the case $\gamma_{1}<0$ when the effective potential is a barrier for an incident soliton. The soliton velocities $v=2 \kappa=0.075$ (dotted line) and $v=2 \kappa=0.05$ (dashed line) correspond to the soliton kinetic energies above and below the potential barrier, respectively.

could consider a soliton with about $10^{3}$ atoms and width $\approx 2 \xi \approx 4 \mu \mathrm{m}$. In experiments we can vary the scattering length by using the Feshbach resonance method by varying in space the external magnetic field $B(x)$ near the resonant value $B_{c}$, such that

$$
a_{s}(x)=a_{b}\left(1-\frac{\Delta}{B_{c}-B(x)}\right),
$$

where $a_{b}$ is the background scattering length and $\Delta$ is the resonance width [27]. Other way to vary the scattering length is the using of the optically induced Feshbach resonances [4-6]. Typical values of variations of the external field $B$ are in the interval $(0-200) \mu \mathrm{m}$ with the period $10 \mu \mathrm{m}$ around the value $352 \mathrm{G}$, where the scattering length has the minimal value $\approx-0.23 \mathrm{~nm}$.

\section{CONCLUSION}

We have investigated reflection, transmission, and trapping of a matter-wave soliton, which is propagating through the interface between a nonlinear uniform media and a nonlinear optical lattice. We study analytically two different limits for broad and narrow soliton dynamics. In both the cases we obtain the characteristics of a soliton trapped by an interface, corresponding to a localized nonlinear surface wave. We derive the effective potentials for the soliton center of mass and the width induced by the joint action of the interface and the media periodic nonlinearity. We obtain the parameters of a localized nonlinear surface state, corresponding 
to a soliton trapped near the interface. Near the stationary point of effective potentials Eqs. (14) and (16), we have calculated frequencies of oscillations of the trapped soliton center and its width. It was also obtained the threshold value of the NOL strength $\gamma_{1}$, separating the transmission and reflection regimes for incident solitons. The predicted surface soliton states can be observed in experiments with BEC in optically induced NOL and in nonlinear optical systems with periodically modulated Kerr nonlinearity.

\section{ACKNOWLEDGMENTS}

The authors are grateful to B. B. Baizakov and E. N. Tsoy for useful discussions. F.Kh.A. is grateful to the grant SAGA Fund 77 by MOSTI for a partial support. M.B. and L.T. thank Fundação de Amparo á Pesquisa do Estado de São Paulo (FAPESP) and Conselho Nacional de Desenvolvimento Científico e Tecnológico $(\mathrm{CNPq})$ for partial support.
[1] A. B. Aceves, J. V. Moloney, and A. C. Newell, Phys. Rev. A 39, 1809 (1989).

[2] Y. S. Kivshar, A. M. Kosevich, and O. A. Chubykalo, Phys. Rev. A 41, 1677 (1990).

[3] Y. V. Kartashov, V. A. Vysloukh, and L. Torner, Opt. Express 14, 1576 (2006).

[4] P. O. Fedichev, Y. Kagan, G. V. Shlyapnikov, and J. T. M. Walraven, Phys. Rev. Lett. 77, 2913 (1996).

[5] F. K. Fatemi, K. M. Jones, and P. D. Lett, Phys. Rev. Lett. 85, 4462 (2000).

[6] M. Theis, G. Thalhammer, K. Winkler, M. Hellwig, G. Ruff, R. Grimm, and J. H. Denschlag, Phys. Rev. Lett. 93, 123001 (2004).

[7] F. Kh. Abdullaev and M. Salerno, J. Phys. B 36, 2851 (2003).

[8] H. Sakaguchi and B. A. Malomed, Phys. Rev. E 72, 046610 (2005).

[9] F. Kh. Abdullaev and J. Garnier, Phys. Rev. A 72, 061605(R) (2005).

[10] G. Theocharis, P. Schmelcher, P. G. Kevrekidis, and D. J. Frantzeskakis, Phys. Rev. A 72, 033614 (2005).

[11] J. Belmonte-Beitia, V. M. Perez-Garcia, V. Vekslerchik, and P. J. Torres, Phys. Rev. Lett. 98, 064102 (2007).

[12] F. K. Abdullaev, A. Gammal, H. L. F. da Luz, and L. Tomio, Phys. Rev. A 76, 043611 (2007).

[13] Y. V. Kartashov, V. A. Vysloukh, and L. Torner, Opt. Lett. 33, 2173 (2008)

[14] Yu. V. Bludov and V. V. Konotop, Phys. Rev. A 74, 043616 (2006).
[15] M. Salerno, V. V. Konotop, and Yu. V. Bludov, Phys. Rev. Lett. 101, 030405 (2008).

[16] F. Kh. Abdullaev, A. Gammal, M. Salerno, and L. Tomio, Phys. Rev. A 77, 023615 (2008).

[17] G. Dong and B. Hu, Phys. Rev. A 75, 013625 (2007).

[18] F. Kh. Abdullaev, A. Gammal, A. M. Kamchatnov, and L. Tomio, Int. J. Mod. Phys. B 19, 3415 (2005).

[19] F. Kh. Abdullaev, A. A. Abdumalikov, and R. M. Galimzyanov, Phys. Lett. A 367, 149 (2007).

[20] G. Fibich, Y. Sivan, and M. Weinstein, Physica D 217, 31 (2006); Y. Sivan, G. Fibich, and M. I. Weinstein, Phys. Rev. Lett. 97, 193902 (2006).

[21] I. L. Garanovich, A. A. Sukhorukov, and Y. S. Kivshar, Phys. Rev. Lett. 100, 203904 (2008).

[22] Y. V. Kartashov, V. A. Vysloukh, A. Szameit, F. Dreisow, M. Heinrich, S. Nolte, A. Tunnrmann, T. Persch, and L. Torner, Opt. Lett. 33, 1120 (2008).

[23] A. Gammal, L. Tomio, and T. Frederico, Phys. Rev. A 66, 043619 (2002); A. Gammal, T. Frederico, and L. Tomio, ibid. 64, 055602 (2001).

[24] D. Anderson, Phys. Rev. A 27, 3135 (1983).

[25] B. A. Malomed, in Progress in Optics, Vol. 43, edited by E. Wolf (Elsevier, Amsterdam, 2002), p. 71.

[26] K. E. Strecker, G. B. Patridge, A. G. Truscott, and R. G. Hulet, Nature (London) 417, 150 (2002); L. Khaykovich, F. Schreck, G. Ferrari, T. Bourdel, J. Cubizolles, L. D. Carr, Y. Castin, and C. Salomon, Science 296, 1290 (2002).

[27] L. P. Pitaevskii and S. Stringari, Bose-Einstein Condensation (Oxford University Press, New York, 2004). 